\title{
Shoreline Changes in Front of New Mansoura City
}

\author{
Hatem Aboelkhair ${ }^{1}$, Atef Ali Qaddah ${ }^{2}$, Magdy Khalil ${ }^{1}$, Ahmed Rakha², Mona Esmail ${ }^{* 2}$ \\ ${ }^{1}$ Geology Department, Faculty of Science, Damietta University, New Damietta, Egypt \\ ${ }^{2}$ Egyptian Environmental Affairs Agency, Egypt
}

Received: 14-08-2019 /Accepted: 14-09-2019

*Corresponding author: monaesmail1989@yahoo.com

\begin{abstract}
The shoreline changes of the coastal zone at New Mansoura City which extends about 27km, have been studied using GIS technique based on RS data. The shoreline spatial and temporal changes in the interval time between years;1984 and 2018 has been estimated. The results show that the coast of New Mansoura City has been affected by morphological changes due to the shoreline accretion and erosion. The area has been subjected to increase in the accretion areas by nearly $2.34 \mathrm{~km}^{2}$, however, the erosional areas reach about $0.195 \mathrm{~km}^{2}$.The average rate of the shoreline accretion area was around $3.12 \mathrm{~m} / \mathrm{y}$, while the rate of the shoreline erosion area was $-1.23 \mathrm{~m} / \mathrm{y}$. Accordingly, the shoreline in the study area is mostly an accretion shoreline and the erosion areas are very limited and should be protected.
\end{abstract}

Keywords: Shoreline changes, Landsat, GIS, Accretion, Erosion, New Mansoura City.

\section{Introduction}

New Mansoura City is located on the northern coastal area of the Nile Delta provenance, $15 \mathrm{~km}$ to the west of Gamasa City. It locates $12 \mathrm{~km}$ to the west of New Damietta City and $20 \mathrm{~km}$ to the east of Baltim City and occupies a length of about $6 \mathrm{~km}$ on the Mediterranean coast (Fig. 1). It is bounded by the latitudes $31^{\circ} 33^{\prime} 15^{\prime \prime} \mathrm{N}$ and $31^{\circ} 25^{\prime} 15^{\prime \prime} \mathrm{N}$, longitudes $31^{\circ} 32^{\prime} 45^{\prime \prime} \mathrm{E}$, and $31^{\circ} 17^{\prime} 45^{\prime \prime} \mathrm{E}$. The city's capacity is expected to be over 1.5 million citizens on an area of 4,000 feddans between Gamasa, Damietta and Kafr El Sheikh cities.

The coastal area is the interface between sea and land and usually displays wide changes in environment and shape because of natural and human activities. Therefore, coastal zone monitoring is an important task in sustainable development and environmental protection.

The rising in sea level and sediments transportation led to erosion and deposition processes which controlled the dynamic changes in the shoreline landforms. Therefore, studying these changes become of a great concern matter. Consequently, it is necessary to perform suitable strategy to maintain the costal resource in order to perform the required sustainable development of coastal areas with respect to industries, agriculture, aquaculture, human settlements and infrastructure.

According to Gens (2010), the vital bases in the coastal zone planning management, sustainable coastal development and environmental protection are; shoreline detection, extraction, and monitoring. The planning of such strategy 
demands the timely detailed information of coastal processes, shoreline conditions, sediment dynamics and hazards.

Over the past three decades, several studies have been conducted to explore the feasibility and accuracy of image analysis applications for monitoring land cover change (Singh 1989, Coppin and Bauer 1996, Mas 1999, Lu et al. 2004) Several change detection techniques using digital images have been reported in the literature (see Singh, 1989 and Lu, et al. 2004). The most common methods applied for change detection include band ratio, band differencing, principal component analysis, vegetation index differencing, and post-classification change detection.

Geographic information system (GIS) and remote sensing (RS) are valuable tools for collection, analysis and interpretation of multiple coastal data. Feng and Han (2012) display that the remotely sensed imagery has been widely used to map shorelines and detect shoreline changes. Saravanan et al. (2015) studied the coastal vulnerability and shoreline extraction via water and land region's automatic extraction techniques. The shoreline change rates can be determined using modern and historic shorelines boundaries, classifications and associated features.

The New Mansoura city coastal zone which extents for a distance of $27 \mathrm{~km}$ has been selected as the area for shoreline change detection in the present study (Fig. 1). The shoreline spatial and temporal changes can be estimated using GIS technique based on RS data.

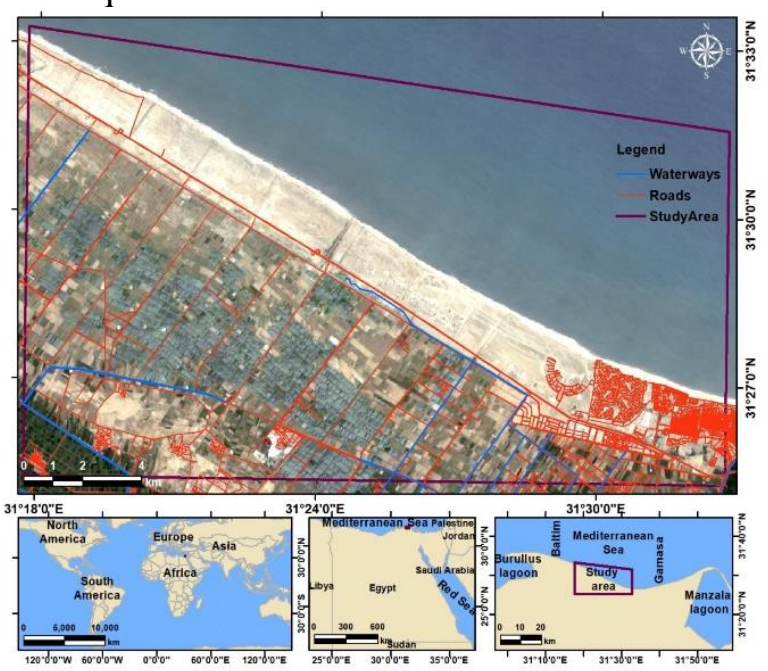

Figure 1: New Mansoura City coastal zone (google earth satellite images) of the study area

\section{Material and Method}

Numerous resources are available for extracting different shoreline positions and quantifying the related changes. GIS can analyze the changes (processes of accretion and erosion) in shoreline by determining the differences between yearly shorelines locations as presented in Fig. (2).

Data of temporal and multispectral satellite images for the years 1984, and 2018 (Landsat Thematic Mapper "TM" and Operational Land Imager (OLI) of $28.5 \times 28.5 \mathrm{~m}$ ground resolution) were processed through ENVI 5.3 image processing software.

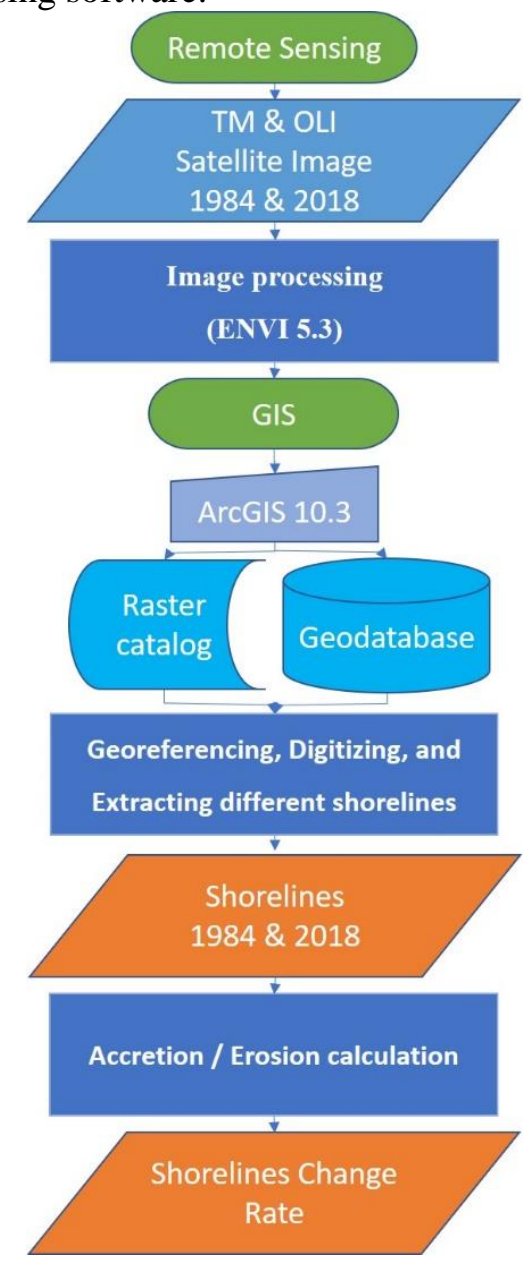

Figure 2: Workflow chart of shoreline changes study methodology

\section{Results}

\section{Shoreline Extraction}

In Arc catalog (via ArcGIS 10.3), Geodatabase containing feature dataset and feature classes were created to evaluate the rate of change for the study area. Feature class (with polyline type) has been 
used to digitize the shoreline for the years 1984 , and 2018 from the satellite images which were spatially re-projected to the Universal Transverse Mercator (UTM 1984). Digitization can easy handling where shoreline features could be recognized from different tones in the sand beach along the dry-wet sand boundaries.

It was relatively easy to distinguish the high-water line on the images as a wet/dry line. Therefore, the shoreline extraction was carried out using "Raster Calculator" (under Map Algebra/Spatial Analyst Tools/Arc Tool Box, via ArcGIS 10.3). In this way, we get a binary image (Fig. 3) with water and land classes only, (where water areas clusters were assigned with pixel value equal one and pixel value equal zero for land areas cluster).

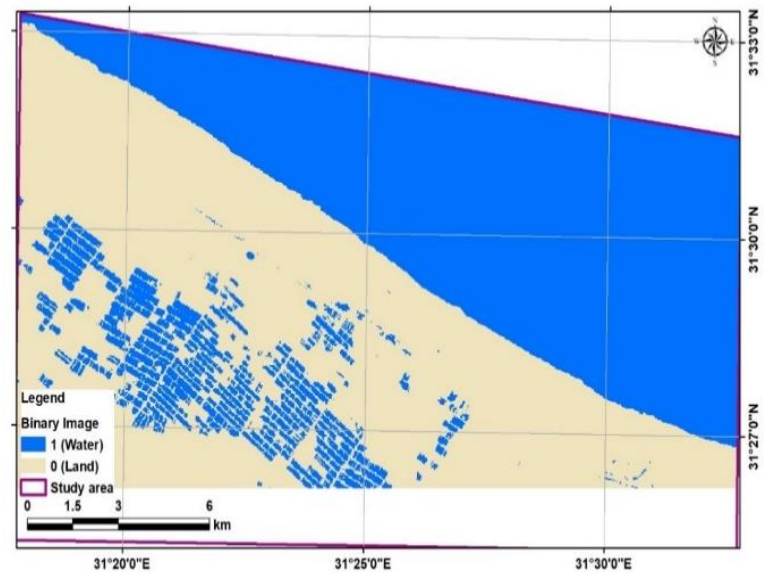

Figure 3: A binary image (water/land classes) with pixel value $1 / 0$, of the coastal zone for the year 2018

\section{Rate of Shoreline Change}

After the shorelines were extracted, the next step is the evaluation of the shoreline changes. Therefore, there is a need to cast the perpendicular transects lines (which were created around at each $200 \mathrm{~m}$ ) on the shorelines to measure the changed distances. The transect-shoreline intersections were consequently used to calculate the rate-ofchange statistics.

\section{Change rate from 1984 to 2018}

During the study period of 1984 to 2018 , the shorelines were extracted, their changes (accretion and erosion) were evaluated, and the rate-ofchange statistics were calculated as shown in Fig. (4) and Tables (1, 2, 3 \& 4).

It has been noticed that, the coast was affected by an increase in the accretion areas by nearly $2.34 \mathrm{~km}^{2}$, where the maximum change in accretion area was around $1.49 \mathrm{~km}^{2}$ and its average change was around $0.23 \mathrm{~km}^{2}$ (Table 1). While the coast defected by lost erosion areas by nearly $0.195 \mathrm{~km}^{2}$, where the maximum change of erosion area was around $0.14 \mathrm{~km}^{2}$ and its average change was around $0.02 \mathrm{~km}^{2}$ (Table 2).

Shoreline accretion area has been observed at an average rate of around $3.12 \mathrm{~m} / \mathrm{y}$ (Tables 3), whereas, a nearly average value of $-1.23 \mathrm{~m} / \mathrm{y}$ was detected for shoreline erosion area (Table 4).

Table (1a): Accretion areas values and its shoreline length changes between years of $1984 \& 2018$.

\begin{tabular}{|c||c|c|c|c|}
\hline $\begin{array}{c}\text { Block } \\
\text { ID }\end{array}$ & $\begin{array}{c}\text { Perimeter } \\
(\mathrm{m})\end{array}$ & $\begin{array}{c}\text { Accretion } \\
\text { Area }\left(\mathrm{m}^{2}\right)\end{array}$ & $\begin{array}{c}\text { 1984 Shore } \\
\text { length }(\mathrm{m})\end{array}$ & $\begin{array}{c}\text { 2018 Shore } \\
\text { length }(\mathrm{m})\end{array}$ \\
\hline \hline 1Acc & 22700.521 & 1485100.990 & 11300.576 & 11269.305 \\
\hline $2 \mathrm{Acc}$ & 218.083 & 416.932 & 109.351 & 108.732 \\
\hline $3 \mathrm{Acc}$ & 240.977 & 1083.144 & 121.900 & 119.078 \\
\hline 4 Acc & 328.619 & 1187.689 & 169.297 & 159.322 \\
\hline 5Acc & 10402.938 & 426770.146 & 5139.813 & 5263.126 \\
\hline 6Acc & 44.401 & 9.289 & 22.129 & 22.273 \\
\hline $7 \mathrm{Acc}$ & 6423.300 & 302880.456 & 3230.958 & 3192.342 \\
\hline $8 \mathrm{Acc}$ & 2034.937 & 42142.192 & 1014.416 & 1020.521 \\
\hline 9Acc & 566.015 & 4423.263 & 281.554 & 284.461 \\
\hline 10Acc & 2676.553 & 75497.439 & 1306.226 & 1370.327 \\
\hline
\end{tabular}

Table (1b): Mini., Max., and Ave. values for different accretion parameter changes between years of $1984 \& 2018$.

\begin{tabular}{|c|c|c|c|}
\hline Parameter & $\begin{array}{c}\text { Minimu } \\
\text { m change }\end{array}$ & $\begin{array}{c}\text { Maximum } \\
\text { change }\end{array}$ & $\begin{array}{c}\text { Average } \\
\text { change }\end{array}$ \\
\hline \hline Perimeter $(\mathrm{m})$ & 44.401 & 22700.521 & 4563.635 \\
\hline Accretion Area $\left(\mathrm{m}^{2}\right)$ & 9.289 & 1485100.990 & 233951.154 \\
\hline 2002 Shore length $(\mathrm{m})$ & 22.129 & 11300.576 & 2269.622 \\
\hline 2018 Shore length $(\mathrm{m})$ & 22.273 & 11269.305 & 2280.949 \\
\hline
\end{tabular}

Table (2a): Erosion areas values and its shoreline length changes between years of 1984 \& 2018 .

\begin{tabular}{|c||c|c|c|c|}
\hline $\begin{array}{c}\text { Block } \\
\text { ID }\end{array}$ & $\begin{array}{c}\text { Perimeter } \\
(\mathrm{m})\end{array}$ & $\begin{array}{c}\text { Erosion } \\
\text { Area }(\mathrm{m} 2)\end{array}$ & $\begin{array}{c}\text { 1984 Shore } \\
\text { length }(\mathrm{m})\end{array}$ & $\begin{array}{c}\text { 2018 Shore } \\
\text { length }(\mathrm{m})\end{array}$ \\
\hline \hline 1Ero & 207.809 & 636.047 & 105.413 & 102.396 \\
\hline 2Ero & 335.690 & 1906.428 & 166.752 & 168.938 \\
\hline 3Ero & 55.016 & 25.782 & 27.656 & 27.359 \\
\hline 4Ero & 42.907 & 51.141 & 22.949 & 19.958 \\
\hline 5Ero & 197.334 & 159.668 & 98.521 & 98.813 \\
\hline 6Ero & 2454.650 & 37691.043 & 1221.595 & 1233.055 \\
\hline 7Ero & 384.927 & 1873.304 & 195.708 & 189.219 \\
\hline 8Ero & 560.095 & 8089.431 & 278.154 & 281.940 \\
\hline 9Ero & 4858.677 & 143398.756 & 2413.918 & 2444.758 \\
\hline 10Ero & 183.420 & 1423.774 & 75.775 & 60.776 \\
\hline
\end{tabular}

Table (2b): Mini., Max., and Ave. values for different erosion parameter changes between years of $1984 \& 2018$.

\begin{tabular}{|l|c|c|c|}
\hline \multicolumn{1}{|c|}{ Parameter } & $\begin{array}{c}\text { Minimum } \\
\text { change }\end{array}$ & $\begin{array}{c}\text { Maximum } \\
\text { change }\end{array}$ & $\begin{array}{c}\text { Average } \\
\text { change }\end{array}$ \\
\hline \hline Perimeter $(\mathrm{m})$ & 42.907 & 4858.677 & 928.052 \\
\hline Erosion Area $\left(\mathrm{m}^{2}\right)$ & 25.782 & 143398.756 & 19525.537 \\
\hline $\begin{array}{l}\text { 2002 Shore length } \\
(\mathrm{m})\end{array}$ & 22.949 & 2413.918 & 460.644 \\
\hline $\begin{array}{l}2018 \text { Shore length } \\
(\mathrm{m})\end{array}$ & 19.958 & 2444.758 & 462.721 \\
\hline
\end{tabular}


Table (3a): Shoreline change rate for accretion areas between years of $1984 \& 2018$.

\begin{tabular}{|c|c|c|}
\hline $\begin{array}{l}\text { Bloc } \\
\text { k ID }\end{array}$ & $\begin{array}{c}\text { Transect } \\
\text { Length }(\mathrm{m})\end{array}$ & $\begin{array}{c}\text { Change } \\
\text { Rate }(\mathrm{m} / \mathrm{y})\end{array}$ \\
\hline $1 \mathrm{Acc}$ & 32.501 & 0.956 \\
\hline $1 \mathrm{Acc}$ & 96.473 & 2.837 \\
\hline $1 \mathrm{Acc}$ & 89.608 & 2.636 \\
\hline $1 \mathrm{Acc}$ & 139.744 & 4.110 \\
\hline $1 \mathrm{Acc}$ & 162.386 & 4.776 \\
\hline $1 \mathrm{Acc}$ & 166.426 & 4.895 \\
\hline $1 \mathrm{Acc}$ & 68.374 & 2.011 \\
\hline $1 \mathrm{Acc}$ & 90.130 & 2.651 \\
\hline $1 \mathrm{Acc}$ & 91.590 & 2.694 \\
\hline $1 \mathrm{Acc}$ & 82.029 & 2.413 \\
\hline $1 \mathrm{Acc}$ & 117.013 & 3.442 \\
\hline $1 \mathrm{Acc}$ & 77.347 & 2.275 \\
\hline $1 \mathrm{Acc}$ & 93.206 & 2.741 \\
\hline $1 \mathrm{Acc}$ & 116.966 & 3.440 \\
\hline $1 \mathrm{Acc}$ & 95.253 & 2.802 \\
\hline $1 \mathrm{Acc}$ & 119.395 & 3.512 \\
\hline $1 \mathrm{Acc}$ & 96.502 & 2.838 \\
\hline $1 \mathrm{Acc}$ & 113.537 & 3.339 \\
\hline $1 \mathrm{Acc}$ & 93.410 & 2.747 \\
\hline $1 \mathrm{Acc}$ & 150.982 & 4.441 \\
\hline $1 \mathrm{Acc}$ & 159.391 & 4.688 \\
\hline $1 \mathrm{Acc}$ & 195.798 & 5.759 \\
\hline $1 \mathrm{Acc}$ & 193.130 & 5.680 \\
\hline $1 \mathrm{Acc}$ & 146.604 & 4.312 \\
\hline $1 \mathrm{Acc}$ & 157.401 & 4.629 \\
\hline $1 \mathrm{Acc}$ & 156.870 & 4.614 \\
\hline $1 \mathrm{Acc}$ & 112.031 & 3.295 \\
\hline $1 \mathrm{Acc}$ & 167.191 & 4.917 \\
\hline $1 \mathrm{Acc}$ & 109.311 & 3.215 \\
\hline $1 \mathrm{Acc}$ & 114.484 & 3.367 \\
\hline $1 \mathrm{Acc}$ & 77.855 & 2.290 \\
\hline $1 \mathrm{Acc}$ & 98.896 & 2.909 \\
\hline $1 \mathrm{Acc}$ & 73.448 & 2.160 \\
\hline $1 \mathrm{Acc}$ & 105.695 & 3.109 \\
\hline $1 \mathrm{Acc}$ & 162.462 & 4.778 \\
\hline $1 \mathrm{Acc}$ & 201.997 & 5.941 \\
\hline $1 \mathrm{Acc}$ & 166.295 & 4.891 \\
\hline $1 \mathrm{Acc}$ & 183.844 & 5.407 \\
\hline
\end{tabular}

\begin{tabular}{|l||c|c|}
\hline $\begin{array}{l}\text { Bloc } \\
\text { I ID }\end{array}$ & $\begin{array}{c}\text { Transect } \\
\text { Length }(\mathrm{m})\end{array}$ & $\begin{array}{c}\text { Change } \\
\text { Rate }(\mathrm{m} / \mathrm{y})\end{array}$ \\
\hline \hline $1 \mathrm{Acc}$ & 176.776 & 5.199 \\
\hline $1 \mathrm{Acc}$ & 177.659 & 5.225 \\
\hline $1 \mathrm{Acc}$ & 185.461 & 5.455 \\
\hline $1 \mathrm{Acc}$ & 210.656 & 6.196 \\
\hline $1 \mathrm{Acc}$ & 178.303 & 5.244 \\
\hline $1 \mathrm{Acc}$ & 183.304 & 5.391 \\
\hline $1 \mathrm{Acc}$ & 175.972 & 5.176 \\
\hline $1 \mathrm{Acc}$ & 155.155 & 4.563 \\
\hline $1 \mathrm{Acc}$ & 139.940 & 4.116 \\
\hline $1 \mathrm{Acc}$ & 113.243 & 3.331 \\
\hline $1 \mathrm{Acc}$ & 127.380 & 3.746 \\
\hline $1 \mathrm{Acc}$ & 127.594 & 3.753 \\
\hline $1 \mathrm{Acc}$ & 152.907 & 4.497 \\
\hline $1 \mathrm{Acc}$ & 166.947 & 4.910 \\
\hline $1 \mathrm{Acc}$ & 189.160 & 5.564 \\
\hline $1 \mathrm{Acc}$ & 163.369 & 4.805 \\
\hline $1 \mathrm{Acc}$ & 152.454 & 4.484 \\
\hline $1 \mathrm{Acc}$ & 123.742 & 3.639 \\
\hline $3 \mathrm{Acc}$ & 15.051 & 0.443 \\
\hline $4 \mathrm{Acc}$ & 11.220 & 0.330 \\
\hline $5 \mathrm{Acc}$ & 5.270 & 0.155 \\
\hline $5 \mathrm{Acc}$ & 49.893 & 1.467 \\
\hline $5 \mathrm{Acc}$ & 119.691 & 3.520 \\
\hline $5 \mathrm{Acc}$ & 85.943 & 2.528 \\
\hline $5 \mathrm{Acc}$ & 57.934 & 1.704 \\
\hline $5 \mathrm{Acc}$ & 128.192 & 3.770 \\
\hline $5 \mathrm{Acc}$ & 66.740 & 1.963 \\
\hline $5 \mathrm{Acc}$ & 80.171 & 2.358 \\
\hline $5 \mathrm{Acc}$ & 98.894 & 2.909 \\
\hline $5 \mathrm{Acc}$ & 121.482 & 3.573 \\
\hline $5 \mathrm{Acc}$ & 90.988 & 2.676 \\
\hline $5 \mathrm{Acc}$ & 153.798 & 4.523 \\
\hline $5 \mathrm{Acc}$ & 181.033 & 5.325 \\
\hline $5 \mathrm{Acc}$ & 155.482 & 4.573 \\
\hline $5 \mathrm{Acc}$ & 105.796 & 3.112 \\
\hline $5 \mathrm{Acc}$ & 111.671 & 3.284 \\
\hline $5 \mathrm{Acc}$ & 38.647 & 1.137 \\
\hline $5 \mathrm{Acc}$ & 64.284 & 1.891 \\
\hline & & \\
\hline
\end{tabular}

\begin{tabular}{|c|c|c|}
\hline $\begin{array}{l}\text { Bloc } \\
k \text { ID }\end{array}$ & $\begin{array}{l}\text { Transect } \\
\text { Length (m) }\end{array}$ & $\begin{array}{c}\text { Change } \\
\text { Rate }(\mathrm{m} / \mathrm{y})\end{array}$ \\
\hline 5 Acc & 73.446 & 2.160 \\
\hline 5 Acc & 36.069 & 1.061 \\
\hline $5 \mathrm{Acc}$ & 71.791 & 2.112 \\
\hline 5 Acc & 87.565 & 2.575 \\
\hline 5 Acc & 50.888 & 1.497 \\
\hline $5 \mathrm{Acc}$ & 96.319 & 2.833 \\
\hline $5 \mathrm{Acc}$ & 55.704 & 1.638 \\
\hline $5 \mathrm{Acc}$ & 69.671 & 2.049 \\
\hline $5 \mathrm{Acc}$ & 25.703 & 0.756 \\
\hline $7 \mathrm{Acc}$ & 13.323 & 0.392 \\
\hline $7 \mathrm{Acc}$ & 135.507 & 3.985 \\
\hline 7Acc & 93.377 & 2.746 \\
\hline $7 \mathrm{Acc}$ & 115.633 & 3.401 \\
\hline $7 \mathrm{Acc}$ & 103.965 & 3.058 \\
\hline $7 \mathrm{Acc}$ & 128.730 & 3.786 \\
\hline 7Acc & 166.934 & 4.910 \\
\hline 7Acc & 189.493 & 5.573 \\
\hline 7Acc & 132.908 & 3.909 \\
\hline $7 \mathrm{Acc}$ & 85.399 & 2.512 \\
\hline $7 \mathrm{Acc}$ & 121.470 & 3.573 \\
\hline $7 \mathrm{Acc}$ & 124.512 & 3.662 \\
\hline $7 \mathrm{Acc}$ & 43.208 & 1.271 \\
\hline $7 \mathrm{Acc}$ & 43.588 & 1.282 \\
\hline $7 \mathrm{Acc}$ & 44.464 & 1.308 \\
\hline 7Acc & 29.541 & 0.869 \\
\hline 8 Acc & 31.043 & 0.913 \\
\hline 8 Acc & 63.054 & 1.855 \\
\hline $8 \mathrm{Acc}$ & 72.043 & 2.119 \\
\hline $8 \mathrm{Acc}$ & 37.405 & 1.100 \\
\hline 8 Acc & 7.472 & 0.220 \\
\hline 9Acc & 23.776 & 0.699 \\
\hline 10 Acc & 55.749 & 1.640 \\
\hline 10Acc & 101.256 & 2.978 \\
\hline $10 \mathrm{Acc}$ & 104.984 & 3.088 \\
\hline $10 \mathrm{Acc}$ & 61.357 & 1.805 \\
\hline $10 \mathrm{Acc}$ & 32.671 & 0.961 \\
\hline $10 \mathrm{Acc}$ & 37.465 & 1.102 \\
\hline 10Acc & 17.250 & 0.507 \\
\hline
\end{tabular}




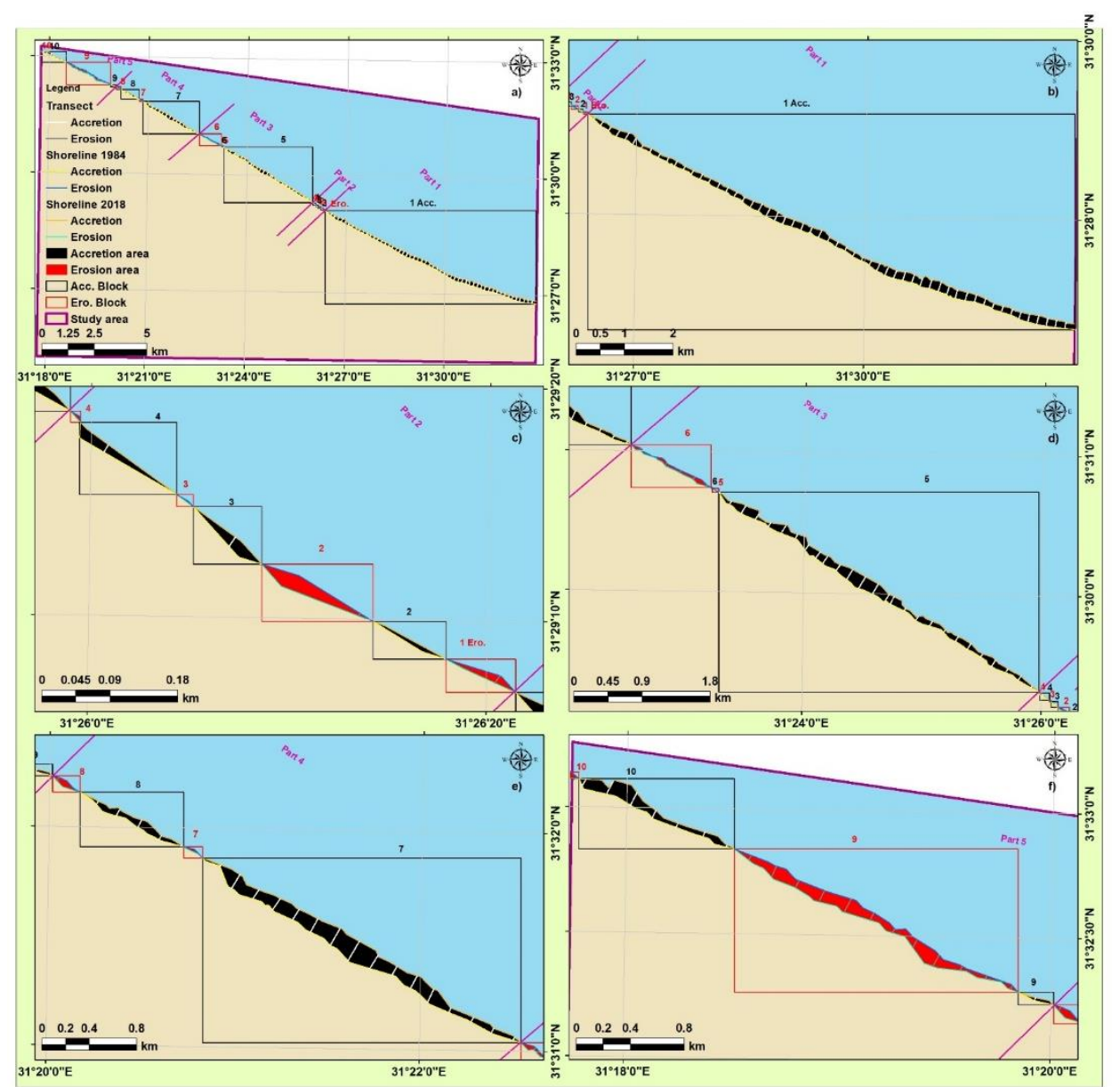

Figure (4): Shoreline changes (accretion and erosion) through years 1984 to 2018: a) the whole shoreline and viewing the blocks of Accretion \& Erosion areas, b) part 1, c) part 2, d) part 3, e) part 4, and f) part 5 of the study area

Table (3b): Mini., Max., and Ave. values of change rate for accretion areas between years of $1984 \& 2018$.

\begin{tabular}{|c|c|c|}
\hline $\begin{array}{c}\text { Mini. Change } \\
\text { Rate }(\mathrm{m} / \mathrm{y})\end{array}$ & $\begin{array}{c}\text { Max. Change } \\
\text { Rate }(\mathrm{m} / \mathrm{y})\end{array}$ & $\begin{array}{c}\text { Ava. Change } \\
\text { Rate }(\mathrm{m} / \mathrm{y})\end{array}$ \\
\hline \hline 0.155 & 6.196 & 3.122 \\
\hline
\end{tabular}

Table (4a): Shoreline change rate values for erosion areas between years of $1984 \& 2018$.

\begin{tabular}{|c|c|c|c|c|c|}
\hline $\begin{array}{c}\text { Block } \\
\text { ID }\end{array}$ & $\begin{array}{l}\text { Transect } \\
\text { Length }(\mathrm{m}) \\
\end{array}$ & $\begin{array}{c}\text { Change } \\
\text { Rate }(\mathrm{m} / \mathrm{y})\end{array}$ & $\begin{array}{c}\text { Block } \\
\text { ID } \\
\end{array}$ & \begin{tabular}{|l|l} 
Transect \\
Length $(\mathrm{m})$ \\
\end{tabular} & $\begin{array}{l}\text { Change } \\
\text { Rate }(\mathrm{m} / \mathrm{y})\end{array}$ \\
\hline 1Ero & 11.606 & -0.341 & 9Ero & 87.934 & -2.586 \\
\hline 2Ero & 3.773 & -0.111 & 9Ero & 57.511 & -1.691 \\
\hline 6Ero & 24.239 & -0.713 & 9Ero & 94.084 & -2.767 \\
\hline 6Ero & 4.771 & -0.140 & 9Ero & 111.254 & -3.272 \\
\hline 6Ero & 42.945 & -1.263 & 9Ero & 60.849 & -1.790 \\
\hline 6Ero & 32.582 & -0.958 & 9Ero & 35.875 & -1.055 \\
\hline 6Ero & 39.768 & -1.170 & 9Ero & 69.227 & -2.036 \\
\hline 6Ero & 17.301 & -0.509 & 9Ero & 94.101 & -2.768 \\
\hline 7Ero & 1.350 & -0.040 & 9Ero & 35.824 & -1.054 \\
\hline 8Ero & 32.970 & -0.970 & 9Ero & 25.507 & -0.750 \\
\hline 8Ero & 4.590 & -0.135 & 9Ero & 31.205 & -0.918 \\
\hline 9Ero & 39.504 & -1.162 & & & \\
\hline
\end{tabular}

Table (4b): Mini., Max., and Ave. values for change rate for erosion areas between years of $1984 \& 2018$.

\begin{tabular}{|c|c|c|}
\hline $\begin{array}{c}\text { Max. Change } \\
\text { Rate }(\mathrm{m} / \mathrm{y})\end{array}$ & $\begin{array}{c}\text { Mini. Change } \\
\text { Rate }(\mathrm{m} / \mathrm{y})\end{array}$ & $\begin{array}{c}\text { Ava. Change } \\
\text { Rate }(\mathrm{m} / \mathrm{y})\end{array}$ \\
\hline \hline-3.272 & -0.040 & -1.226 \\
\hline
\end{tabular}

\section{Conclusions}

The results of the present Study show that the New Mansoura city coastal zone during the period of 1984 2018 has affected by morphological changes due to the shoreline changes (such as accretion and erosion). The area has been affected by an increase of the accretional areas by nearly $2.34 \mathrm{~km}^{2}$ and defected by lost erosional areas by nearly $0.195 \mathrm{~km}^{2}$. Whereas the shoreline accretional zone has been observed with an average rate of around $3.12 \mathrm{~m} / \mathrm{y}$ and a nearly average value of $-1.23 \mathrm{~m} / \mathrm{y}$ has been detected for shoreline erosional zone.

Accordingly, the shoreline in the study area is mostly an accretional shoreline and the erosional areas which may need protection are very limited. 


\section{References}

Coppin, P.R. and Bauer, M.E. (1996), Digital change detection in forest ecosystems with remote sensing imagery. Remote Sensing Reviews, Vol. 13, p. 207-234.

Feng, Y., and Han, Z., (2012), Cellular automata approach to extract shoreline from remote sensing imageries and its application. J Image Graph 17(3), 441 - 446.

Gens, R., (2010), Remote sensing of coastlines: detection extraction and monitoring. International Journal of Remote Sensing. 31(7), pp. 1819 - 1836.
Lu, D., Mausel. P., Brondiozio, E. and Moran, E. (2004), Change detection techniques. Int J Rem Sens 25: p. 2365-2407.

Mass, J.F. (1999), Monitoring land-cover changes: a comparison of change detection techniques. Int $\mathbf{J}$ Remote Sens Vol. 20: p.139-152.

Saravanan. S., Parthasarathy K.S.S., Kumaresan.P.R., Vishnu prasath.S.R., Vasanth Kumar.T. (2015), Shoreline Change Detection for Chennai Coast Using Geospatial Techniques. International ITT Roorkee, India. 17 -19 December.

Singh, A. (1989), Digital change detection techniques using remotely sensed data. International Journal of Remote Sensing, Vol. 10, p.989-1003.

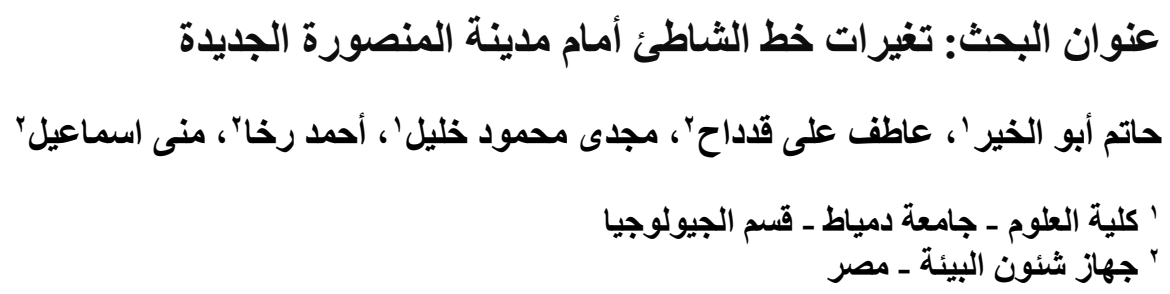

الملخص العربي

تقع مدينة المنصورة الجديدة على ساحل البحر الأبيض المتوسط بين مدينتي بلطيم وجمصه (حو الى باني آكم غرب مدينة جمصده) .

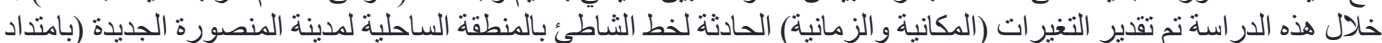

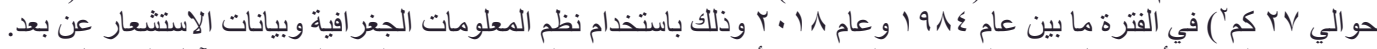

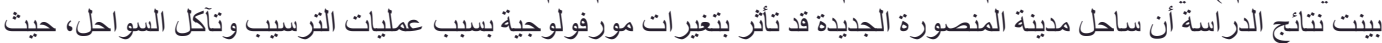

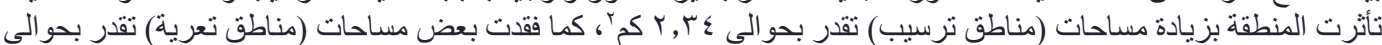

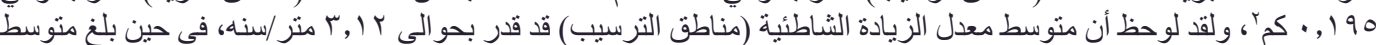

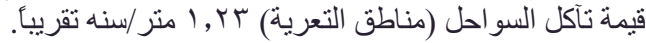
ومن هذه الدر اسة يمكن اعتبار ساحل منطقة الدر اسة في مجمله يتميز بترسيبات شاطئية وبعض مناطق تعرية قليلة قد تحتاج إلى حماية محدودة هذه الإنة 